\title{
The Cross-Cultural Adaptation and the Reliability Test for the Chinese-Version Dietary Behavior and Psychological Series Scales in Maintenance Hemodialysis Patients
}

\section{Xue Li ${ }^{\prime}$ \\ Yan Shan ${ }^{2}$ \\ Yajing Gao' \\ Xinxin Jiang ${ }^{\prime}$ \\ Hong Wang' \\ Xuzhen Yang' \\ Yabo Ding'}

'Nursing and Health School of Zhengzhou University, Zhengzhou, Henan Province, People's Republic of China; ${ }^{2}$ Medical School of Zhengzhou University, Zhengzhou, Henan Province, People's Republic of China
Correspondence: Yan Shan Email syl10@sina.com
Purpose: To translate three novel measures of psychological mechanisms associated with dietary behavior, including the Dietary Goal-Desire Incongruence scale (DG-DI), the Motivation for Dietary Self-control scale (MDSC), the Satisfaction with Dietary Behavior scale (SWDB), to cross-culturally adapt the measures into Chinese and verify their reliability and validity in maintenance hemodialysis patients.

Method: After the forward translation and the back-translation, the perspective of a panel of experts and cognitive interviews with maintenance hemodialysis patients were used to ensure cultural relevance of the three scales. Subsequently, 420 maintenance hemodialysis patients from three hemodialysis centers in Zhengzhou were recruited for the item analysis and the internal consistency, content validity, construct validity and reliability tests.

Results: The moderate associations between items and domains $(r>0.50)$ and the significant differences between the high and low groups were measured by an independent sample $t$ test $(\mathrm{P}<0.001)$. The Cronbach's $\alpha$ coefficient of the DG-DI reached 0.884 . The Cronbach's $\alpha$ of the MDSC was 0.831 , with Cronbach's $\alpha$ values $(0.865,0.800$ and 0.797 for "Internal", "External" and "Amotivation", respectively). In addition, the Cronbach's alpha of the SWDB was 0.914 . The scale-level content validity index (S-CVI) reached $0.96,0.98$ and 1.00 for the DG-DI, the MDSC and the SWDB, respectively. The exploratory factor analysis verified the scale structures of five factors, and the cumulative variance contribution rate of the respective factor was $65.507 \%$. The confirmatory factor analysis was conducted to test the original structure of the scale.

Conclusion: The DG-DI, the MDSC and the SWDB showed satisfactory psychometric characteristics. They could effectively assess the eating behavior of hemodialysis patients. Subsequent studies should recruit other different population samples in China to verify the applicability of the scale.

Keywords: dietary behavior, reliability, validity, cultural adaptation, dietary compliance

\section{Introduction}

Over the past few decades, the incidence of chronic kidney disease has been rising year by year, which turns out to be a major global public health challenge. ${ }^{1,2}$ As the disease progresses, chronic kidney disease is capable of developing into end-stage kidney diseases if it is not effectively treated and controlled, which requires renal replacement therapy (dialysis or renal Transplantation, RRT) 
to maintain normal function. ${ }^{3}$ As indicated by one existing study, the number use of RRT worldwide is estimated to be more than doubled by $2030 .{ }^{4}$ To be specific, over $1,000,000$ patients have received hemodialysis each year globally, and hemodialysis is the most extensively used, safe and effective renal replacement therapy. 5,6

Hemodialysis is a long-term treatment therapy. To prevent the slowing progression of the disease, patients require long-term strict self-management, among which dietary acts as a vital factor. ${ }^{7}$ Maintenance hemodialysis (MHD) patients with reasonable dietary behavior are capable of lowering the incidence of complications and mortality, thereby improving their quality of life and survival. ${ }^{8}$ However, as reported in existing studies, MHD patients exhibit poor dietary behaviors (eg, decreased dietary management ability and poor dietary compliance), ${ }^{9}$ thereby causing poor control of liquid intake, ${ }^{10}$ involuntarily excessive intake of food with high phosphorus and potassium, ${ }^{11}$ as well as a low self-health awareness. ${ }^{12}$ As a result, the quality of dialysis treatments is affected, and the deterioration of the condition accelerating. ${ }^{13}$ In China, $50 \%$ of MHD patients have dietary nonadherence behaviors, and the situation of individual dietary control behavior is not optimistic. ${ }^{14}$ Accordingly, the adverse eating behavior of MHD patients should be assessed and improved to reduce relevant complications and improve the quality of life of patients.

Thus far, most of the studies on the diet of hemodialysis patients focused on dietary compliance, nutritional status, and intake of high phosphorus and high potassium without any in-depth exploration of the psychological factors of patients' eating behavior. ${ }^{15,16}$ The eating behavior measurement tools in China have been imported and revised from abroad (eg, the Three-factor Eating Questionnaire (TFEQ) of Stunkard, ${ }^{17}$ the Dutch Eating Behavior Questionnaire(DEBQ) of van Strien, ${ }^{18}$ as well as the Emotional Eating Scale(EES) ${ }^{19}$ ). The assessment tools for diet measurement in patients with renal disease are mostly The Renal Adherence Attitudes Questionnaire (RAAQ) and The Renal Adherence Behavior Questionnaire (RABQ). ${ }^{20,21}$ Rare studies have been conducted to identify the psychological factors associated with individuals' controlling eating behaviors, and effective scales have been rare to measure the problems of individuals' eating self-control.

Dietary control in hemodialysis patients is impacted by various factors, ${ }^{22,23}$ and an insight into the factors involved in food choices can promote healthy changes in eating behavior. Eating behavior is influenced by emotional regulation, and the analysis of the different psychological and social motivations determining people's eating patterns can facilitate interventions that promote disease control or treatment to be implemented. ${ }^{24,25}$ The Dietary Goal-Desire Incongruence scale (DG-DI), the Motivation for Dietary Self-control scale (MDSC), and the Satisfaction with Dietary Behavior scale (SWDB) were created by Hamish to validate three novel psychological mechanism assessment tools associated with eating behavior. $^{26}$ The DG-DI assesses the degree to which an individual's ideal eating behavior conflicts with their actual desired goals. In addition, the MDSC consists of three components assessing the degree and type of motivation an individual has in controlling eating behavior. Moreover, the SDWB acts as a Diet-Related Behavioral Satisfaction Scale, ie, an individual assessment of the consistency of eating behavior with healthy eating goals.

Existing studies on the diet of MHD patients have not explored their psychological aspects. This series of questionnaires have been applied to the MHD population to analyze the internal factors of the occurrence of poor eating behaviors in the relevant patients and improve the ability to self-control eating. Accordingly, this study aimed to translate the three questionnaires into Chinese and assess their psychometric characteristics, as an attempt to provide a novel perspective for Chinese clinical patients to assess eating behavior.

\section{Methods}

\section{Measures}

The permission to use the DG-DI, the MDSC and the SWDB was obtained from the author (Navjot Bhullar) under a license agreement from the University of New England. Specific to the respective scale, answer with a 7-point Richter scale, where $0=$ does not describe me at all, and $6=$ expresses me completely. The DG-DI refers to a 1 -factor scale with 6 items; the higher the scores, the greater the incongruity in eating goals and desires, which are associated with more uncontrolled and emotional eating. The MDSC consists of 11 items in three domains: internal motivation, external motivation, and amotivation, high scores on internal and external motivation for dietary self-control scores associated with high levels of dietary restraint, as well as high scores on an amotivation subscale correlated with high levels of apathy. The SWDB with 1-factor comprises 8 items; higher scores indicate the 
more satisfied you are with self-eating behavior. Love et al demonstrated that the internal consistency assessed by Cronbach's alpha $(\alpha)$ was $0.94,0.78$ and 0.94 for the three scales, respectively. ${ }^{26}$ Furthermore, the confirmatory factor analyses supported the model fit of each scale's factor solution.

\section{Translation and Cross-Cultural Adaptation}

The author of the three scales was contacted by email. The authorization was obtained, and then the three scales were translated into Chinese under Brislin's translation guidelines. $^{27,28}$ Phase I of the translation and crosscultural adaptation of the DG-DI, the MDSC and the SWDB was performed in the following steps: (1) Forward translation: the scales were translated into Chinese by two researchers proficient in English and Chinese. The two researchers consisted of a nursing specialist knowledgeable about hemodialysis, as well as a specialist with expertise in English. Subsequently, our research team (ie, 1 professor, 1 doctor and 2 postgraduates majoring in Dialysis care) compared the two Chinese versions and engaged in the discussions to produce the final version. (2) Back translation: The translation was independently translated into English by two other bilingual researchers. One was a $\mathrm{PhD}$ candidate familiar with nursing knowledge and having been a visiting student in the United States for one year, and the other in charge of College English courses was not familiar with medical professional knowledge. Our research team compared the two translated versions, analyzed the similarities and differences between them and provided the feedback to the original author for the verification. (3) Cultural adaptation: Five experts were invited, and they engaged in the study to verify whether the concepts and connotations of the original scale were retained. The expert group comprised two first-line nurse managers with expertise in dialysis and dietary care, two professors with psychological nursing experience, as well as a clinician with more than 15 years of experience in dialysis. The experts were asked to check whether the meaning of the Chinese version was consistent with that of the original version by complying with their professional knowledge and clinical work experience, as well as to assess the cultural relevance of the Chinese version. (4) Content validity: This expert group was asked to use a four-point scale from 1 (not relevant) to 4 (highly relevant), to assess the three scales' item content effectiveness index (I-CVI; ie, the number of experts with a score of 3 or 4 on each item divided by the total number of experts) and scale content effectiveness index (S-CVI; ie, the number of items rated as 3 or 4 by all experts as a percentage of total items). An item with I-CVI and S-CVI values of $>0.78$ and $>0.80$ or more was considered valid. ${ }^{29,30}$ (5) A face-to-face cognitive interview was conducted among 10 patients on the long-term hemodialysis treatment recruited from dialysis centers to assess the insights into these three questionnaires. To further confirm whether the problems in the cognitive interview have been addressed, a pilot trial was conducted on 30 MHD patients to verify whether the contents of the three scales for measuring eating behavior were easily understood, and patients' recommendations were recorded and carried out their final modifications to the Chinese version of the DG-DI, the MDSC and the SWDB based on their feedback.

In the Phase II, the following contents were used for psychometric validation of the three scales: (a) item analysis; (b)construct validity; (c) internal consistency reliability; (d) test-retest reliability; (e) split-half reliability; (f) Content Validity; and (g) Reliability validity. The collected data were randomly divided into two groups using SPSS21.0. A total of 420 data of MHD patients were collected in this study. One group included 210 cases for exploratory factor analysis (EFA), and the other group included 210 cases for confirmatory factor analysis(CFA). We used Guttman split-half reliability and test-retest reliability to evaluate the stability of the three scales. Two weeks after the data collection, 30 MHD patients volunteered for the second survey to verify the test-retest reliability.

\section{Questionnaire Distribution}

We calculated the required sample size based on the ratio of the study subjects to the number of items $(5: 1-10: 1),{ }^{31}$ the DG-DI, the MDSC and the SWDB with 25 statements. In this study, a total of $420 \mathrm{MHD}$ patients were collected and randomly assigned to two groups with SPSS21.0. The first group consisted of 210 patients for the exploratory factor analysis (EFA), and the other 210 patients pertained to the second group for the confirmatory factor analysis (CFA). The following inclusion criteria were applied: 1) aged $\geq 18$ years (According to the age range of young in the Working Law of the people's Republic of China); 2) Diagnosed as a maintenance hemodialysis patient in line with K/DOQI guidelines; 3) Regular hemodialysis 
treatment $\geq 3$ months; 4) Ability to communicate and willing to participate in the study. Patients suffered from severe mental or cognitive diseases were excluded. Potential participants were recruited through convenience sampling, which were sampled in two hemodialysis centers in Zhengzhou from November 2020 to March 2021.

For all participants, the general information was collected, including age, sex, marital status, educational level, monthly household income, place of residence, work status, dialysis status, living status, medical insurance, as well as types of complications. Since dialysis patients were on long-term and regular dialysis, it could be convenient to assess the test-retest reliability. 30 patients from those who completed the initial survey were randomly selected, and they were asked to complete a second questionnaire two weeks later.

\section{Statistical Analysis}

IBM SPSS Statistics 21 (SPSS) and IBM SPSS AMOS 26.0 (AMOS) were adopted to conduct the Statistical analyses. The demographic characteristics (eg, the numbers, frequencies, means and standard deviations (SDs)) of the participants were analyzed by using descriptive statistical methods. The association between the items and the total score was assessed by using Pearson correlation analysis and critical ratio (CR), the item-total associations above 0.3 and the $\mathrm{CR}$ reaching over 0.4 were considered adequate. $^{32}$ The internal consistency of the three scales was measured based on the alpha value, with an alpha value for the whole scale and each domain $\geq 0.7$ as the minimum acceptable value. ${ }^{33,34}$

EFA and CFA were used to test the construct validity in the Chinese version of the DG-DI, the MDSC and the SWDB. In the measurement of EFA, the Kaiser - Meyer Olkin (KMO) and Bartlett's Sphericity tests were employed, and factors with a factor load $>0.4$ and the eigenvalue $>1.0$ were extracted. Subsequently, AOMS26.0 was used to further verify the preset model of the scales. The criteria below were assessed: (1) chisquare values divided by the degrees of freedom ( $\chi 2 / \mathrm{df})$ between 1and 3 to be considered acceptable; (2) the root mean square error of approximation (RMSEA) of $<0.08$; (3) the goodness-of-fit index (GFI) $>0.90$; (4) normed fit index (NFI), adjusted GFI (AGFI) and comparative fit index (CFI), all $>0.90 .^{35}$ Cronbach's Alpha was used to assess the internal consistency reliability of the Chinese version of the DG-DI, the MDSC and the SWDB, with $\geq 0.7$ as the minimum acceptable value. ${ }^{36,37}$ Specific to the reliability of the retest, the intra-group correlation coefficient (ICC) was measured for the total score of the three scales and the scores of each domain.

\section{Ethical Considerations}

The license was obtained from the original author to translate the DG-DI, the MDSC and the SWDB into Chinese and use them. This study was approved by the Ethics Committee of Zhengzhou University, China. The head of the dialysis center, all researchers and participants signed the informed consent before the investigation. We actively briefed participants on the purpose and significance of the study, and they were assured that their answers would be anonymous, and their personal information would be confidential until they signed the informed consent. This study was reviewed by the Ethics Committee of Zhengzhou University (Access Number: ZZUIRB 2021-47).

\section{Results}

\section{Translation and Adaptation}

A high degree of consistency was identified between our back translation and the original version. The translators and experts consulted only revised a few expressions about cultural differences and semantic accuracy. For instance, the "appetite" in the first entry of the DG-DI was in the back, and the translated version was inconsistent with the "strong urge" in the original scale. After the discussion, we decided not to use the word appetite directly. When experts consulted on cultural adaptation, different experts gave different explanations to the translation of "I cannot trust my food cravings to guide me to healthy food choices". Moreover, as indicated from the results of pilot trials, some assessors did not understand the meaning of this clause during the assessment. Thus, the sentence order was adjusted through the group discussion and combined with the opinions of experts and assessors to make it easier to understand. Since the body image of MHD patients was changed due to the edema of excessive fluid and salt intake, and there was the situation that they would not be satisfied with their own image in the media. The sixth item of the MDSC was also applicable to MHD people, and the item was retained.

\section{Study Participants}

On the whole, 445 patients receiving maintenance hemodialysis were approached, and 420 of them (94.3\%) signed the informed consent and completed the assessment of all the 
scales. Table 1 lists the demographic and medical characteristics of the 420 respondents. Participants were aged from 21 to 86 years, with an average age of 50.26 years (SD: 14.259). The average age of the 10 cognitive interview participants was 43.2 years old (SD: 9.76). The participants were primarily male $(80 \%), 20 \%$ had primary education, and $80 \%$ had high school education or above. The average age of the 30 pilot participants reached 48.14 years (SD: 14.882). The participants were largely male (76.7\%), and $73.3 \%$ had received a high school education or above.

\section{Item Analysis}

The item analysis was conducted on the three scales of the DG-DI, the MDSC and the SWDB, respectively, and the results are listed in Tables 2 and 3. As indicated from the results, there was a positive association between each item and its domain, and all the item-total associations were above 0.5 . Given the total score, a total of 420 valid samples were ranked from the highest to the lowest. The first $27 \%$ were classified as high, and the last $27 \%$ were low. ${ }^{32}$ The item differences between the high and low groups were tested by performing the independent sample $\mathrm{T}$ tests. As revealed from the result, all CR reached over $0.3(\mathrm{~T}>4, \mathrm{P}<0.001)$, which explained the items of the three scales had high discrimination.

\section{Content Validity}

Validity refers to the extent to which a measurement or test a scale is intended to measure the psychological structure, in which content validity acts as an important indicator of the usefulness and relevance of the assessment tool. ${ }^{32}$ As suggested from the content validity analysis results, the I-CVI of the DG-DI, the MDSC and the SWDB ranged from 0.80 to 1.00 , and the S-CVI of the three scales reached $0.967,0.981$ and 1.00 , respectively. The mentioned result exceeded the recommended levels of 0.78 and 0.80 , respectively, which verified the sufficient content validity of the three scales.

\section{Construct Validity}

EFA (with a sample of 210) presents a five-factor model, taking up $65.507 \%$ of the total variance, with eigenvalues $>1(5.031,3.798,2.825,2.549$ and 2.173). Table 4 elucidates the items loaded on the mentioned five factors. The Kaiser-Meyer-Olkin (KMO) coefficient reached 0.868, the Bartlett's test was significant $(\mathrm{P}<0.001)$, which indicated that our data were suitable for EFA. Subsequently,
Table I Demographic Characteristics of Participants $(n=420)$

\begin{tabular}{|c|c|}
\hline Characteristics & n (\%) \\
\hline Age (years), mean $\pm S D$ & $50.26 \pm 14.259$ \\
\hline \multicolumn{2}{|l|}{ Gender } \\
\hline Male & $256(60.8)$ \\
\hline Female & $164(39.2)$ \\
\hline \multicolumn{2}{|l|}{ Marital status } \\
\hline Unmarried & $41(9.8)$ \\
\hline Married & $347(82.6)$ \\
\hline Married, widowed or divorced & $32(7.6)$ \\
\hline \multicolumn{2}{|l|}{ Educational level } \\
\hline Primary school or less & $63(15.0)$ \\
\hline Middle school & $102(24.3)$ \\
\hline High school technical secondary school & $106(25.2)$ \\
\hline College degree or above & $149(35.5)$ \\
\hline \multicolumn{2}{|l|}{ Employment status } \\
\hline Employed & $109(26.0)$ \\
\hline Unemployed or Retired & $311(74.0)$ \\
\hline \multicolumn{2}{|l|}{ Income per month (RMB) } \\
\hline$<1000$ & $28(6.7)$ \\
\hline $1000 \sim 3000$ & $136(32.4)$ \\
\hline $3000 \sim 5000$ & $155(36.9)$ \\
\hline$>5000$ & $101(24.0)$ \\
\hline \multicolumn{2}{|l|}{ Residence } \\
\hline Rural area & $58(13.8)$ \\
\hline Villages and towns area & $41(9.8)$ \\
\hline Urban area & $321(76.4)$ \\
\hline \multicolumn{2}{|l|}{ Medical insurance } \\
\hline Urban employee medical insurance & $103(24.5)$ \\
\hline Urban and rural residents' medical insurance & $177(42.1)$ \\
\hline Rural residents'Medical insurance & $134(31.9)$ \\
\hline Other or no insurance & $6(1.4)$ \\
\hline \multicolumn{2}{|l|}{ Dialysis duration } \\
\hline$\leq 1$ year & $72(17.1)$ \\
\hline I 5 year & $221(52.6)$ \\
\hline$>5$ year & $127(30.2)$ \\
\hline \multicolumn{2}{|l|}{ Types of complications } \\
\hline 0 & $67(16.0)$ \\
\hline I & $216(51.4)$ \\
\hline 2 & $99(23.6)$ \\
\hline$\geq 3$ & $38(9.0)$ \\
\hline \multicolumn{2}{|l|}{ Primary caregiver } \\
\hline Spouse & $257(6 \mid .2)$ \\
\hline Child & $38(9.0)$ \\
\hline Parents & $53(12.6)$ \\
\hline Others & $72(17.1)$ \\
\hline
\end{tabular}

Abbreviation: $S D$, standard deviation. 
Table 2 Item Score and Correlation Coefficient with the Domain

\begin{tabular}{|c|c|c|}
\hline Item & $\begin{array}{l}\text { Correlation Coefficient with } \\
\text { the Domain (R) }\end{array}$ & $\begin{array}{l}\text { Score } \\
\text { (Mean } \pm \text { SD) }\end{array}$ \\
\hline DG-DI I & $0.86 I^{* *}$ & $4.75 \pm 0.976$ \\
\hline DG-DI 2 & $0.847^{* *}$ & $4.81 \pm 1.047$ \\
\hline DG-DI 3 & $0.90 I^{* *}$ & $4.53 \pm 1.015$ \\
\hline DG-DI 4 & $0.918^{* *}$ & $5.18 \pm 0.804$ \\
\hline DG-DI 5 & $0.919 * *$ & $4.81 \pm 1.021$ \\
\hline DG-DI 6 & $0.919 * *$ & $4.73 \pm 0.865$ \\
\hline MDSC I & $0.797^{* *}$ & $5.36 \pm 1.057$ \\
\hline MDSC 2 & $0.728 * *$ & $5.25 \pm 1.118$ \\
\hline MDSC 3 & $0.80 I^{* *}$ & $5.05 \pm 1.055$ \\
\hline MDSC 4 & $0.834 * *$ & $5.10 \pm 1.089$ \\
\hline MDSC 5 & $0.759 * *$ & $4.50 \pm 1.305$ \\
\hline MDSC 6 & 0.580 ** & $3.39 \pm 1.697$ \\
\hline MDSC 7 & $0.78 I^{* *}$ & $5.54 \pm 1.074$ \\
\hline MDSC 8 & $0.608^{* *}$ & $4.45 \pm 1.421$ \\
\hline MDSC 9 & $0.569 * *$ & $5.75 \pm 1.166$ \\
\hline MDSC 10 & $0.648^{* *}$ & $6.30 \pm 1.174$ \\
\hline MDSC II & $0.629 * *$ & $5.82 \pm 1.314$ \\
\hline SWDB I & $0.821^{* *}$ & $4.64 \pm 1.130$ \\
\hline SWDB 2 & $0.794^{* *}$ & $4.91 \pm 1.110$ \\
\hline SWDB 3 & $0.84 I^{* *}$ & $4.95 \pm 0.994$ \\
\hline SWDB 4 & $0.853^{* *}$ & $4.67 \pm 1.180$ \\
\hline SWDB 5 & $0.858^{* *}$ & $4.82 \pm 0.998$ \\
\hline SWDB 6 & $0.908^{* *}$ & $5.11 \pm 1.025$ \\
\hline SWDB 7 & $0.919 * *$ & $5.15 \pm 0.943$ \\
\hline SWDB 8 & $0.908 * *$ & $5.17 \pm 0.940$ \\
\hline
\end{tabular}

Note: **Indicates $\mathrm{P}<0.01$.

Abbreviation: SD, standard deviation.

we performed the factor model validation on the 25 items of the tool (with another sample of 210), the identical structure and structure were kept as the default model $(\mathrm{CMIN} / d f=2.092 ; \mathrm{RMSEA}=0.072 ; \mathrm{GFI}=0.822 ; \mathrm{NFI}$ $=0.846 ;$ TLI $=0.901 ;$ CFI $=0.912$, see Table 5). Accordingly, all the items in the analysis were retained.

\section{Reliability Analysis}

The internal consistency and inter-rating reliability of the three scales were summarized. The Cronbach's alpha of our DG-DI for MHD patients reached 0. 884. The Cronbach's $\alpha$ coefficient of the MDSC was calculated as 0.831 , with Cronbach's $\alpha$ values reaching $0.865,0.800$ and 0.797 for "Internal", "External" and "Amotivation", respectively. In addition, the Cronbach's alpha of the SWDB was 0.914. The split-half coefficients of the DGDI, the MDSC and the SWDB reached 0.883,0.702 and 0.891 , respectively. For DG-DI, the retest reliability of the
MDSC and the SWDB were 0.835, 0.841 and 0.760, respectively. As suggested from the mentioned results, the three scales exhibited high reliability.

\section{Discussion}

The DG-DI, the MDSC and the SWDB are 7-point Richter scales, where $0=$ does not describe me at all and $6=$ describes me completely. In the process of translation and debugging, to promote better calculation and assessment of the results, the scoring method was modified from 0 to 6 points to 1 to 7 points, as an attempt to more effectively boost the use of this series of scales in China.

Our research results show that cross-cultural adjustment and translation did not change the original structures and domains of the DG-DI, the MDSC and the SWDB, but only some items were worded to make them better understood in the Chinese context. Suggestions from the panelists helped revise the content, suggesting that sentences that were not smooth should be changed and that the expression of "desire" should be understood as appetite, to better measure eating behavior. In the assessment of content validity, the I-CVI values of all items meet the standard. The expert group and participants of cognitive interview considered that the mentioned 25 items are not inconsistent with the Chinese cultural background, which can effectively understand and facilitate the measurement of the eating behavior of different groups, and assess the variations of individual eating behavior. Thus, in this process, only part of the translation of the sentence has been adjusted, and no items were deleted.

The item analysis method was employed to conduct the independent sample $T$ test between the high-level group and the low-level group. According to the $\mathrm{T}$ value arranged in the Table 3 , each item was $>4 \quad(\mathrm{P}<0.001)$, with a range of 7.986 29.833. Accordingly, a difference was considered to exist between the high group and the low group (the difference was statistically significant). Moreover, the association between each item and the total score was calculated in SPSS, and the Pearson $\mathrm{R}$ value of each item was greater than 0.3 . For this reason, this item is considered to effectively represent the topic of this field. Thus, all the items were saved during this process.

In the development of the scale, the original author measured the original scale structure of the three scales of the DG-DI, the MDSC and the SWDB, respectively. According to the results of the mediation analysis test, the 
Table 3 Independent Sample $t$ Test for the High Scoring and Low Scoring Group

\begin{tabular}{|c|c|c|c|c|c|c|c|}
\hline \multirow[t]{2}{*}{ Item } & \multirow[t]{2}{*}{ CR (tvalue) } & \multirow[t]{2}{*}{ df } & \multirow[t]{2}{*}{$\mathbf{P}$} & \multirow[t]{2}{*}{ Mean Difference } & \multirow[t]{2}{*}{ Std. Error Difference } & \multicolumn{2}{|c|}{ 95\% Confidence Interval of the Difference } \\
\hline & & & & & & Lower & Upper \\
\hline DG-DI I & 20.179 & 226 & $<0.001$ & 2.447 & 0.121 & 2.208 & 2.686 \\
\hline DG-DI 2 & 18.393 & 226 & $<0.001$ & 2.456 & 0.134 & 2.193 & 2.719 \\
\hline DG-DI 3 & 24.452 & 226 & $<0.001$ & 2.693 & 0.110 & 2.476 & 2.910 \\
\hline DG-DI 4 & 29.833 & 226 & $<0.001$ & 3.000 & 0.101 & 2.802 & 3.198 \\
\hline DG-DI 5 & 26.123 & 226 & $<0.001$ & 2.930 & 0.112 & 2.709 & 3.151 \\
\hline DG-DI 6 & 26.695 & 226 & $<0.001$ & 2.649 & 0.099 & 2.454 & 2.845 \\
\hline MDSC I & 16.470 & 226 & $<0.001$ & 2.298 & 0.140 & 2.023 & 2.573 \\
\hline MDSC 2 & 15.454 & 226 & $<0.001$ & 2.254 & 0.146 & 1.967 & 2.542 \\
\hline MDSC 3 & 16.931 & 226 & $<0.001$ & $2.28 I$ & 0.135 & 2.015 & 2.546 \\
\hline MDSC 4 & 17.852 & 226 & $<0.001$ & 2.421 & 0.136 & 2.154 & 2.688 \\
\hline MDSC 5 & 14.852 & 226 & $<0.001$ & 2.263 & 0.152 & 1.963 & 2.563 \\
\hline MDSC 6 & 7.986 & 226 & $<0.001$ & 1.439 & 0.180 & 1.084 & 1.794 \\
\hline MDSC 7 & 15.912 & 226 & $<0.001$ & 2.377 & 0.149 & 2.083 & 2.672 \\
\hline MDSC 8 & 9.127 & 226 & $<0.001$ & 1.649 & 0.181 & 1.293 & 2.005 \\
\hline MDSC 9 & 9.305 & 226 & $<0.001$ & $1.56 \mathrm{I}$ & 0.168 & 1.231 & 1.892 \\
\hline MDSCIO & 10.328 & 226 & $<0.001$ & 1.807 & 0.175 & 1.462 & 2.152 \\
\hline MDSC I I & $10.64 \mid$ & 226 & $<0.001$ & 1.904 & 0.179 & $1.55 \mathrm{I}$ & 2.256 \\
\hline SWDB I & 14.977 & 226 & $<0.001$ & 2.061 & 0.138 & 1.790 & 2.333 \\
\hline SWDB 2 & 11.830 & 226 & $<0.001$ & 1.640 & 0.139 & 1.367 & 1.914 \\
\hline SWDB 3 & 13.518 & 226 & $<0.001$ & $1.78 \mid$ & 0.132 & 1.521 & 2.040 \\
\hline SWDB 4 & 17.456 & 226 & $<0.001$ & 2.439 & 0.140 & 2.163 & 2.714 \\
\hline SWDB 5 & $|3.68|$ & 226 & $<0.001$ & 1.737 & 0.127 & 1.487 & 1.987 \\
\hline SWDB 6 & 18.392 & 226 & $<0.001$ & 2.272 & 0.124 & 2.029 & 2.515 \\
\hline SWDB 7 & 20.388 & 226 & $<0.001$ & 2.272 & 0.112 & 2.052 & 2.492 \\
\hline SWDB 8 & 20.472 & 226 & $<0.001$ & 2.474 & 0.121 & 2.236 & 2.712 \\
\hline
\end{tabular}

Abbreviations: $\mathrm{CR}$, critical ratio; df, degree of freedom; Std, standard.

total motivation for dietary self-control was found to have a mediating relationship with goal-desire conflict and eating behavior satisfaction. ${ }^{26}$ Accordingly, we tested the structural validity of the three scales together. According to the factor structure of the original scale, we preset a five-factor model, and the data are well adapted to the model, providing sufficient positive evidence for the effectiveness of the construction. In the meantime, EFA results classified 25 items into 5 factors, and the results of the original scale were consistent, taking up $65.507 \%$ of the total variance. The loading coefficients of all items were large $>0.4$, which showed that these results were ideal.

Reliability analysis showed satisfactory internal and inter-score consistency. Cronbach alpha values of the three scales ranged from 0.800 to 0.914 , which was basically consistent with the results of the original version. The split reliability of the MDSC is 0.702 , showing slightly lower reliability, which is consistent with the results of the original scale. Moreover, in terms of testretest reliability, the SWDB is the unstable one among the three scales, which may be affected by changes in the quality of life and unstable self-assessment of MHD patients. Thus, further reliability measurement of the three scales is needed in the future.

\section{Implications for Research and Practice}

Diet is an indispensable part of hemodialysis patients, especially its role in the progression of the disease. Promoting and maintaining good dietary behavior for a long time can effectively improve the quality of life of MHD patients. Some studies ${ }^{38,39}$ highlighted that a better understanding and assessment of an individual's mental and emotional state of eating is critical to promoting healthy changes in eating behavior. This study introduced and translated the DG-DI, the MDSC and the SWDB scales. The cultural adjustment was conducted on the translated version, and the scales were applied to MHD patients to explore their reliability and validity, as an attempt to provide an effective tool from a novel perspective for the assessment of eating behaviors in Chinese 
Table 4 Factor Structure of DG-DI, MDSC and SWDB

\begin{tabular}{|c|c|c|c|c|c|}
\hline \multirow[t]{2}{*}{ Item } & \multicolumn{5}{|c|}{ Derived Factors } \\
\hline & Factor-I & Factor-2 & Factor-3 & Factor-4 & Factor-5 \\
\hline I often feel proud of my food choices. & 0.637 & & & & \\
\hline I wish my eating patterns were healthier. & 0.659 & & & & \\
\hline I am satisfied with the amount of food that I eat. & 0.710 & & & & \\
\hline There is no need to change anything about my current dietary behavior. & 0.720 & & & & \\
\hline I believe my current diet is nutritious. & 0.780 & & & & \\
\hline I am satisfied with the types of food that I eat. & 0.838 & & & & \\
\hline My diet makes me feel good. & 0.825 & & & & \\
\hline I am satisfied with my current eating behavior. & 0.791 & & & & \\
\hline $\begin{array}{l}\text { When I think about some foods I get a strong urge to eat them, even if I am not } \\
\text { hungry. }\end{array}$ & & 0.734 & & & \\
\hline I cannot trust my food cravings to guide me to healthy food choices. & & 0.692 & & & \\
\hline I find it tiring to struggle against my cravings. & & 0.747 & & & \\
\hline I feel tempted by unhealthy food. & & 0.793 & & & \\
\hline I often experience internal conflict when I am thinking about what to eat. & & 0.804 & & & \\
\hline $\begin{array}{l}\text { I love certain foods so much that it is hard to resist eating them even when they } \\
\text { are not in-line with my goals. }\end{array}$ & & 0.734 & & & \\
\hline I try to avoid eating some foods because I want to be healthier. & & & 0.803 & & \\
\hline I try to eat more healthy foods because I feel good when I eat that way. & & & 0.780 & & \\
\hline I have strong motivation to control what I eat. & & & 0.705 & & \\
\hline I enjoy being in control of my eating patterns. & & & 0.712 & & \\
\hline I try to control what I eat so that other people will think well of me. & & & & 0.784 & \\
\hline $\begin{array}{l}\text { I want to control my eating because the images I see in the media make me feel } \\
\text { unattractive. }\end{array}$ & & & & 0.646 & \\
\hline I try to watch what I eat because of doctor recommendations. & & & & 0.667 & \\
\hline I try to resist some foods because of pressure from people around me. & & & & 0.795 & \\
\hline Improving my diet is a low priority in my life right now. & & & & & 0.770 \\
\hline I do not see any reasons to control my eating. & & & & & 0.802 \\
\hline I do not try to resist tempting food. & & & & & 0.754 \\
\hline Eigenvalue & 5.031 & 3.798 & 2.825 & 2.549 & 2.173 \\
\hline Cumulative variance & 20.126 & 35.318 & 46.618 & 56.814 & 65.507 \\
\hline
\end{tabular}

patients. Second, though further verification of this series of scales is needed in the future, the three scales can be

Table 5 Goodness-of-Fit Indices for the DG-DI, MDSC and SWDB Factor Models

\begin{tabular}{|l|l|l|}
\hline Index & Value & Reference Standard \\
\hline $\mathrm{x}^{2} / \mathrm{df}$ & 2.092 & $\mathrm{I} \sim 3$ \\
Goodness of fit index (GFI) & 0.822 & Close to I \\
Normed-fit index (NFI) & 0.846 & Close to I \\
Comparative fit index (CFI) & 0.912 & Close to I \\
TLI & 0.901 & Close to I \\
RMSEA estimate & 0.072 & $<0.08$ \\
\hline
\end{tabular}

applied to a wider range of people, especially patients with chronic diseases. For instance, the MDSC can be adopted to develop an intervention study on the intrinsic motivation of diabetic patients to control the intake of refined carbohydrates. Besides, the DG-DI can be used to predict or assess the changes in the attitude of obese patients to control diet. It is particularly important to improve and control dietary behaviors in the long-term rehabilitation process. Subsequently, the three scales could act as the result measurement of individuals' eating behaviors, and they could predict individuals' eating behavior problems in accordance with the results. The greater the conflict between eating goals and desires, the less the motivation 
and the less the restraint ability to eat would be. Lastly, the three scales exhibited the favorable psychometrical properties that could act as a potential psychological mechanism between intervention and outcome measures, helping to develop new, more targeted interventions to improve poor eating behaviors and health outcomes in different groups.

\section{Strengths and Limitations}

Our translation follows the international standards, and we have a panel of experts to ensure the content of our cultural adaptation. At the same time, part of the target population is invited for cognitive interviews and experiments, and appropriate psychometric tests are accepted. However, there are several limitations to our study. First, our samples were only collected from three hemodialysis centers in Zhengzhou, China through convenience sampling, so the samples in the study were not representative enough. The eating behavior of MHD patients who come from remote areas such as towns or who receive less education on eating health remains unclear. The diversity of samples is beneficial to enrich the assessment content of tools and improve the translation quality of tools. Accordingly, the reliability and validity test with a large sample size is required subsequently. Second, in our study, standards-related validity was verified to assess the structural scale of the DG-DI, the MDSC and the SWDB scales. Subsequent work should use relevant tools to verify the criterion-related validity of the three scales.

\section{Conclusion}

The DG-DI, the MDSC and the SWDB scales have been translated from the English version into Chinese and crosscultural adapted. The results of this study show that this tool has good internal consistency and reliability, suitable content, and exhibits a similar factor structure with the original English version. It is an effective tool to assess the psychological factors of eating behavior of Chinese patients. Though further verification and testing are needed, the three scales can be actively promoted to assess the eating behavior problems of different populations and promote the implementation of intervention.

\section{Acknowledgments}

We would like to thank all the participants, researchers, specialists and hemodialysis center workers for their contributions to this work. We express our gratitude to the authors of the scale for allowing us to translate and use them.

\section{Author Contributions}

All authors contributed to data analysis, drafting or revising the article, have agreed on the journal to which the article will be submitted, gave final approval of the version to be published, and agree to be accountable for all aspects of the work.

\section{Disclosure}

The authors declare no conflicts of interest in this study.

\section{References}

1. Hill NR, Fatoba ST, Oke JL, et al. Global prevalence of chronic kidney disease - a systematic review and meta-analysis. PLoS One. 2016;11(7):e158765. doi:10.1371/journal.pone.0158765

2. Webster AC, Nagler EV, Morton RL, et al. Chronic kidney disease. Lancet. 2017;389(10075):1238-1252. doi:10.1016/S0140-6736(16) 32064-5

3. Tandukar S, Palevsky PM. Continuous renal replacement therapy: who, when, why, and how. Chest. 2019;155(3):626-638. doi:10.1016/ j.chest.2018.09.004

4. Liyanage $T$, Ninomiya $T$, Jha $\mathrm{V}$, et al. Worldwide access to treatment for end-stage kidney disease: a systematic review. Lancet. 2015;385 (9981):1975-1982. doi:10.1016/S0140-6736(14)61601-9

5. Shinkawa H, Yasunaga H, Hasegawa K, et al. Mortality and morbidity after pancreatoduodenectomy in patients undergoing hemodialysis: analysis using a national inpatient database. Surgery. 2019;165 (4):747-750. doi:10.1016/j.surg.2018.10.009

6. Zhang D. Establishment of National Hemodialysis Case Information Registration System and Analysis of the Treatment of Anemia in Hemodialysis Patients. The PLA Military Medical Training College; 2012.

7. Maraj M, Kusnierz-Cabala B, Dumnicka P, et al. Malnutrition, Inflammation, Atherosclerosis Syndrome (MIA) and diet recommendations among end-stage renal disease patients treated with maintenance hemodialysis. Nutrients. 2018;10:1. doi:10.3390/nu10010069

8. Fotheringham J, Fogarty DG, El NM, et al. The mortality and hospitalization rates associated with the long interdialytic gap in thrice-weekly hemodialysis patients. Kidney Int. 2015;88 (3):569-575. doi:10.1038/ki.2015.141

9. Clark S, Farrington K, Chilcot J. Nonadherence in dialysis patients: prevalence, measurement, outcome, and psychological determinants. Semin Dial. 2014;27(1):42-49. doi:10.1111/sdi.12159

10. Nadri A, Khanoussi A, Hssaine Y, et al. Effect of a hemodialysis patient education on fluid control and dietary. Nephrol Ther. 2020;16 (6):353-358. doi:10.1016/j.nephro.2020.03.011

11. Rizk R, Karavetian M, Hiligsmann M, et al. Effect of stage-based education provided by dedicated dietitians on hyperphosphataemic haemodialysis patients: results from the nutrition education for management of osteodystrophy randomised controlled trial. J Hum Nutr Diet. 2017;30(5):554-562. doi:10.1111/jhn.12472

12. Rhee CM, Ahmadi SF, Kovesdy CP, et al. Low-protein diet for conservative management of chronic kidney disease: a systematic review and meta-analysis of controlled trials. $J$ Cachexia Sarcopenia Muscle. 2018;9(2):235-245. doi:10.1002/jcsm.12264

13. Lambert K, Mullan J, Mansfield K. An integrative review of the methodology and findings regarding dietary adherence in end stage kidney disease. BMC Nephrol. 2017;18(1):318. doi:10.1186/s12882017-0734-Z

14. Yinxia Z, Yingxia W, Guiying $C$, et al. Investigation and analysis of nutritional status and influencing factors of maintenance hemodialysis patients. Chinese J Nurs Manage. 2014;14(03):169-170. 
15. Garcia-Llana H, Remor E, Selgas R. Adherence to treatment, emotional state and quality of life in patients with end-stage renal disease undergoing dialysis. Psicothema. 2013;25(1):79-86.

16. Clark-Cutaia MN, Ren D, Hoffman LA, et al. Adherence to hemodialysis dietary sodium recommendations: influence of patient characteristics, self-efficacy, and perceived barriers. J Ren Nutr. 2014;24 (2):92-99. doi:10.1053/j.jrn.2013.11.007

17. Stunkard AJ, Messick S. The three-factor eating questionnaire to measure dietary restraint, disinhibition and hunger. J Psychosom Res. 1985;29(1):71-83. doi:10.1016/0022-3999(85)90010-8

18. van Strien T, Ouwens MA. Effects of distress, alexithymia and impulsivity on eating. Eat Behav. 2007;8(2):251-257. doi:10.1016/j. eatbeh.2006.06.004

19. Arnow B, Kenardy J, Agras WS. The Emotional Eating Scale: the development of a measure to assess coping with negative affect by eating. Int J Eat Disord. 1995;18(1):79-90. doi:10.1002/1098-108X (199507)18:1<79::AID-EAT2260180109>3.0.CO;2-V

20. Rushe H, Mcgee HM. Assessing adherence to dietary recommendations for hemodialysis patients: the Renal Adherence Attitudes Questionnaire (RAAQ) and the Renal Adherence Behaviour Questionnaire (RABQ). J Psychosom Res. 1998;45(2):149-157. doi:10.1016/S0022-3999(97)00228-6

21. Gao Y, Shan Y, Jiang T, et al. Dietary adherence, self-regulatory fatigue and trait self-control among chinese patients with peritoneal dialysis: a cross-sectional study. Patient Prefer Adherence. 2021;15:443-451. doi:10.2147/PPA.S298231

22. Ahrari S, Moshki M, Bahrami M. The relationship between social support and adherence of dietary and fluids restrictions among hemodialysis patients in Iran. J Caring Sci. 2014;3(1):11-19.

23. Mellon L, Regan D, Curtis R. Factors influencing adherence among Irish haemodialysis patients. Patient Educ Couns. 2013;92(1):88-93. doi:10.1016/j.pec.2013.01.023

24. Cardoso AP, Ferreira V, Leal M, et al. Perceptions about healthy eating and emotional factors conditioning eating behaviour: a study involving Portugal, Brazil and Argentina. Foods. 2020;9:9. doi:10.3390/foods9091236

25. Brug J, Kremers SP, Lenthe F, et al. Environmental determinants of healthy eating: in need of theory and evidence. Proc Nutr Soc. 2008;67(3):307-316. doi:10.1017/S0029665108008616

26. Love H, Bhullar N, Schutte NS. Psychological aspects of diet: development and validation of three measures assessing dietary goal-desire incongruence, motivation, and satisfaction with dietary behavior. Appetite. 2019;138:223-232. doi:10.1016/j.appet.2019.03.018
27. Sousa VD, Rojjanasrirat W. Translation, adaptation and validation of instruments or scales for use in cross-cultural health care research: a clear and user-friendly guideline. J Eval Clin Pract. 2011;17 (2):268-274. doi:10.1111/j.1365-2753.2010.01434.x

28. Jones PS, Lee JW, Phillips LR, et al. An adaptation of Brislin's translation model for cross-cultural research. Nurs Res. 2001;50 (5):300-304. doi:10.1097/00006199-200109000-00008

29. Lynn MR. Determination and quantification of content validity. Nurs Res. 1986;35(6):382-385. doi:10.1097/00006199-19861100 0-00017

30. Polit DF, Beck CT. The content validity index: are you sure you know what's being reported? Critique and recommendations. Res Nurs Health. 2006;29(5):489-497. doi:10.1002/nur.20147

31. Shultz KS, Whitney DJ, Mj Z. Measurement Theory in action:Case Studies and Exercises. New York: Routledge; 2013.

32. Wu ML. Questionnaire Statistical Analysis Practice: SPSS Operation and Application. Chongqing: Chongqing University Press; 2019.

33. Barbaranelli C, Lee CS, Vellone E, et al. The problem with Cronbach's Alpha: comment on Sijtsma and van der Ark (2015). Nurs Res. 2015;64(2):140-145. doi:10.1097/NNR.00000000000 00079

34. Bentler PM. Alpha, dimension-free, and model-based internal consistency reliability. Psychometrika. 2009;74(1):137-143. doi:10.10 07/s11336-008-9100-1

35. Kim H, Ku B, Kim JY, et al. Confirmatory and exploratory factor analysis for validating the phlegm pattern questionnaire for healthy subjects. Evid Based Complement Alternat Med. 2016;2016:26 96019.

36. Spiliotopoulou G. Reliability reconsidered: cronbach's alpha and paediatric assessment in occupational therapy. Aust Occup Ther J. 2009;56(3):150-155. doi:10.1111/j.144-1630.2009.00785.x

37. Bujang MA, Omar ED, Baharum NA. A review on sample size determination for Cronbach's alpha test: a simple guide for researchers. Malays J Med Sci. 2018;25(6):85-99.

38. Paans N, Gibson-Smith D, Bot M, et al. Depression and eating styles are independently associated with dietary intake. Appetite. 2019;134:103-110. doi:10.1016/j.appet.2018.12.030

39. Ferrao AC, Correia P, Ferreira M, et al. Perceptions towards healthy diet of the portuguese according to area of work or studies. Zdr Varst. 2019;58(1):40-46.

\section{Publish your work in this journal}

Patient Preference and Adherence is an international, peer-reviewed, open access journal that focusing on the growing importance of patient preference and adherence throughout the therapeutic continuum. Patient satisfaction, acceptability, quality of life, compliance, persistence and their role in developing new therapeutic modalities and compounds to optimize clinical outcomes for existing disease states are major areas of interest for the journal. This journal has been accepted for indexing on PubMed Central. The manuscript management system is completely online and includes a very quick and fair peer-review system, which is all easy to use. Visit http:// www.dovepress.com/testimonials.php to read real quotes from published authors. 